Rabaska

Revue d'ethnologie de l'Amérique française

\title{
Choisir son identité culturelle : le cas d'Allan/Alain Kelly
}

\section{Ronald Labelle}

Numéro 1, 2003

URI : https://id.erudit.org/iderudit/201601ar

DOI : https://doi.org/10.7202/201601ar

Aller au sommaire du numéro

Éditeur(s)

Société québécoise d'ethnologie

ISSN

1703-7433 (imprimé)

1916-7350 (numérique)

Découvrir la revue

Citer cet article

Labelle, R. (2003). Choisir son identité culturelle : le cas d'Allan/Alain Kelly.

Rabaska, (1), 11-20. https://doi.org/10.7202/201601ar d'utilisation que vous pouvez consulter en ligne.

https://apropos.erudit.org/fr/usagers/politique-dutilisation/ 


\title{
Choisir son identité culturelle : le cas d'Allan/Alain Kelly
}

\author{
RonALD LABELLE
}

Université de Moncton

En 2001, j'ai présenté à l'université Laval une thèse de doctorat portant sur le témoignage autobiographique d'Allan ou Alain Kelly, un Acadien de descendance à la fois française et irlandaise. Malgré le fait que ce dernier écrivait son prénom «Allan», les francophones de sa famille et de son entourage l'ont toujours appelé « Alain», et c'est ainsi que je préfère le désigner. La thèse que je lui ai consacrée avait pour but de démontrer qu'à travers l'étude d'un récit de vie, on peut apprendre comment la perception de soi-même et de son milieu de vie est influencée par les croyances religieuses et la vision du surnaturel que possède un individu.

Dans mon analyse d'un corpus constitué des résultats d'une centaine d'heures d'entrevues menées sur une période de vingt ans, j'ai songé d'abord à examiner la question de l'identité culturelle d'Alain Kelly. Il me paraissait qu'un témoignage autobiographique extrêmement détaillé, provenant d'un individu bilingue et biculturel, pourrait présenter une excellente occasion pour étudier des cultures en contact. Un premier article sur le sujet a d'ailleurs été réalisé en collaboration avec Marge Steiner. Cet article intitulé « The French Irishman as Cultural Broker : An Acadian at the Miramichi Folksong Festival ${ }^{1}$ » décrivait la façon dont Alain Kelly arrivait à présenter des chansons acadiennes dans le contexte d'un festival qui célèbre l'héritage culturel anglophone de la Miramichi. Nous y avons décrit monsieur Kelly comme un chanteur folklorique acadien qui, à cause de son caractère bilingue, pouvait adapter ses performances à un public anglophone.

Lorsque je me suis intéressé à l'ensemble des modes d'expression culturelle d'Alain Kelly, j'ai constaté qu'il pouvait jouer le rôle d'interlocuteur non seulement comme chanteur mais aussi comme raconteur. Connaissant bien les coutumes, croyances et légendes qui circulaient en milieu acadien pendant la première moitié du XXe siècle, sa familiarité avec la culture populaire des anglophones du Nouveau-Brunswick lui permettait d'adapter ses récits à

1. Voir Northeast Folklore - Essays in Honor of Edward D. Ives, Pauleena MacDougall et David Taylor (dir.), Orono, University of Maine Press, 2000, pp. 97-112. 
l'anglais ${ }^{2}$. La question du caractère biculturel d'Alain Kelly a toutefois été écartée dans l'élaboration de ma thèse de doctorat puisque ce dernier m'apparaissait comme un Acadien avant tout, malgré son bilinguisme et ses origines mixtes. J'aimerais revenir sur cette question ici, puisque, par son itinéraire de vie, Alain Kelly nous permet d'observer des cultures en contact. Fredrik Barth avait démontré que la diversité culturelle persiste non seulement quand différents groupes sont isolés les uns des autres, mais que des facteurs sociaux çontribuent à maintenir les frontières entre ces derniers, même dans des cas où deux groupes sont en relation d'interdépendance ${ }^{3}$. La question que je me pose est donc de savoir si un individu qui hérite de deux langues et de deux cultures et qui vit dans un milieu où deux communautés vivent côte à côte ne se voit pas dans l'obligation de se ranger d'un côté ou de l'autre de la frontière culturelle.

Alain Kelly est né à Pointe-Sapin sur la côte est du Nouveau-Brunswick en 1903. Il était le onzième enfant de William Kelly et de Judith Robichaud. Pointe-Sapin était un village entièrement francophone situé non loin de communautés anglophones comme Baie-du-Vin, St. Margaret's et Kouchibougouac. William Kelly, le seul anglophone à Pointe-Sapin, était justement originaire de la petite communauté catholique et irlandaise de Kouchibougouac. Quant à Judith Robichaud, elle est née à Pointe-Sapin, où sa famille était établie depuis longtemps, mais sans toutefois faire partie du groupe de familles qui possédaient la plupart des terres dans le voisinage.

Alors qu'Alain Kelly était jeune enfant, la famille a été éprouvée à deux reprises. D'abord, les Kelly ont été expulsés de la demeure et de la terre qu'ils occupaient, à cause d'un conflit avec le propriétaire pour qui travaillait William Kelly. Ensuite, Judith Robichaud-Kelly est décédée, laissant plusieurs enfants en bas âge. Alain est donc allé vivre chez un marchand nommé Hubert Robichaud qui demeurait à l'autre extrémité du village. Entre l'âge de 5 ans et de 12 ans environ, il a donc vécu au sein d'une famille francophone où il a reçu une éducation catholique qui le marquera pour le reste de sa vie. Il commente ainsi l'influence qu'a eue sur lui la famille Robichaud:

C'est là [chez les Robichaud] que j'ai appris le peu que je sais. [...] Il faut croire que le Bon Dieu m'a conduit là, moi, il y a pas moyen! Parce qu'il y en a pas un chez nous qui sait lire ni rien de ça ${ }^{4}$.

C'est là qu'il a aussi été témoin de plusieurs pratiques traditionnelles qui accompagnaient les fêtes du calendrier religieux. Par exemple, on pratiquait

2. Voir Doug Underhill, Miramichi Tales, tall \& true, Saint-Jean, N.-B., Neptune Publishing, 1999.

3. Fredrik Barth, «Introduction », Ethnic Groups and Boundaries, Bergen et Oslo, Universitets Forlaget, 1969, pp. 9-10.

4. CÉA, coll. R. Labelle, enreg. 3464 (1998). 
encore à Pointe-Sapin la coutume de « chanter les Alléluia ». Des groupes de paroissiens se rendaient à la porte de chaque maison du village pendant la nuit du samedi saint en chantant: Si vous dormez, réveillez-vous/Notre Seigneur est ressuscité/En Galilée, vous le verrez/Alléluia/Alléluia, Alléluia, Alléluias.

Le jour de la Fête-Dieu, on se levait tôt le matin pour recueillir des gouttes de la rosée que l'on croyait être bénéfique pour la santé. Alain Kelly raconte que certains se roulaient dans l'herbe le matin pour que la rosée les imprègne. De même, on recueillait la neige qui tombait au début du mois de mai, le «mois de Marie », pour plus tard se frotter les yeux endoloris avec l'eau provenant de cette neige ${ }^{6}$.

En plus de connaître de telles coutumes traditionnelles pendant son enfance à Pointe-Sapin, Alain Kelly a aussi été plongé dans le monde du surnaturel, vivant dans un milieu où l'on croyait aux revenants, aux apparitions du diable et aux feux-follets. Il se rappelle, par exemple, que les feux-follets apparaissaient souvent sur un certain rocher au bord de la mer. Une lumière, qui se déplaçait sur le rocher, faisait peur aux habitants.

Chez Hubert Robichaud, Alain parlait anglais seulement le dimanche lorsque son père lui rendait visite. Parvenu à l'adolescence, il est retourné vivre avec son père et ses frères, après quoi une grave infection au pied l'a obligé à passer plusieurs mois à l'Hôtel-Dieu de Chatham. Par la suite, il a logé pendant quelques années chez un entrepreneur forestier anglophone dans le village de Barnaby River.

Quand il a commencé sa vie active à l'âge de 16 ans, Alain Kelly était parfaitement bilingue, il avait vécu à différents moments dans des milieux francophones et anglophones et il partait alors pour travailler dans les chantiers forestiers où il aurait pu s'associer autant à un groupe linguistique qu'à l'autre. Il a ensuite passé des hivers entiers dans les chantiers où une quarantaine d'hommes vivaient ensemble complètement coupés du monde extérieur.

Parmi ces travailleurs, il y avait autant d'anglophones unilingues originaires de la Miramichi que de francophones unilingues venant du nordest de la province. Alain Kelly était bien placé pour jouer le rôle d'intermédiaire, aussi bien entre les deux groupes linguistiques qu'entre les bûcherons acadiens et leurs patrons anglophones. Il défendait les intérêts des francophones, par exemple en vérifiant les factures rédigées en anglais que ces derniers recevaient des magasins des compagnies forestières, pour s'assurer qu'on ne les trichait pas.

5. CÉA, coll. R. Labelle, enreg. 403 (1979).

6. CÉA, coll. R. Labelle, ms. 54 (1980). 
Kelly aurait pu profiter de son bilinguisme pour obtenir la faveur des patrons anglophones dans les camps forestiers. Pour montrer qu'il était solidaire des bûcherons acadiens, il raconte qu'un automne un groupe de jeunes bûcherons du nord de la province étaient arrivés aux chantiers en disputant contre le patron et les conditions de travail. Reconnaissant le mot boss, le patron voulait savoir si les hommes parlaient contre lui, mais Kelly l'a rassuré en lui disant que ses compagnons trouvaient qu'ils avaient un bon boss $^{7}$.

Les bûcherons francophones appréciaient la présence d'Alain Kelly, non seulement parce qu'il leur servait d'interprète, mais aussi parce qu'il les divertissait avec ses chansons. Il dit :

Ah, moi, ils étiont tout le temps après moi. Je savais un lot de chansons. Tous les soirs, ils étiont après moi dans les bunks pour chanter ${ }^{8}$.

Bien que la chanson traditionnelle fût un passe-temps important, autant pour les bûcherons anglophones que pour les francophones, Alain Kelly semble avoir surtout chanté avec ces derniers, puisqu'il se rappelle surtout avoir appris des chansons françaises dans les chantiers.

Kelly entretenait de bons rapports avec les deux groupes de bûcherons, mais il s'identifiait plutôt aux Acadiens, avec lesquels il avait plus d'affinités. Il explique cela en disant simplement : «Moi, je me tenais quasiment tout le temps avec les Français. Il me semble que ça allait mieux 9 . » Cela lui a valu le surnom French Irishman de la part des bûcherons anglophones. Il aurait été difficile pour lui de ne pas s'identifier à l'un des deux groupes, étant donné qu'il existait une séparation linguistique informelle dans les chantiers forestiers. On peut supposer qu'ayant été élevé dans le village acadien de Pointe-Sapin, Alain Kelly se sentait chez lui parmi des francophones surtout. Le fait que plusieurs travailleurs anglophones étaient de religion protestante pouvait aussi créer un obstacle à son intégration parmi eux, parce qu'il avait reçu une éducation catholique qui marquait son identité. Il attachait une grande importance à la prière et sa vision du monde était liée de près à une conception du surnaturel profondément catholique.

Une fois qu'il a commencé à gagner sa vie, Alain Kelly n'est plus retourné vivre à Pointe-Sapin, mais passait les mois d'été dans les scieries et les usines de bois à pâte de la Miramichi. Là encore, il vivait dans un milieu à caractère anglophone, mais il s'associait surtout à des travailleurs francophones. C'est là aussi qu'il a rencontré sa future épouse, Léontine Doiron, une Acadienne de Tracadie qui travaillait comme domestique à Newcastle. Après leur mariage

\footnotetext{
7. CÉA, coll. R. Labelle, enreg. 3406 (1998).

8. CÉA, coll. R. Labelle, enreg. 1626 (1983).

9. CÉA, coll. R. Labelle-J. McGinity, enreg. 322 (1981).
} 
en 1924, le couple est allé vivre pendant plusieurs années dans la région de Tracadie, au coeur de la Péninsule acadienne.

À Tracadie, Alain Kelly s'engageait comme ouvrier agricole lorsqu'il n'était pas dans les chantiers forestiers. Il avait alors comme projet d'obtenir sa propre terre agricole et c'est en 1934 qu'il obtint une concession de terre dans la partie nord du district de Beaver Brook, dans la Miramichi. Là, Kelly est devenu encore une fois un intermédiaire entre les deux groupes linguistiques. La majorité des colons à Beaver Brook étaient francophones, mais ils étaient rattachés à la municipalité de Newcastle et ils devaient communiquer avec les autorités anglophones pour obtenir de l'aide matérielle pendant la crise économique ou encore pour régler des questions reliées aux concessions de terres. De plus, les commerçants locaux, les acheteurs de bois et les représentants du ministère provincial des Terres étaient tous anglophones.

Un climat de méfiance existait entre les colons et les divers intervenants anglophones à Beaver Brook. Alain Kelly jouait souvent le rôle de porteparole, surtout pour les nombreux parents de son épouse Léontine qui se trouvaient dans la localité. Plusieurs personnes dépendaient de son aide, puisque les colons de Beaver Brook étaient complètement démunis, n'ayant aucun revenu et étant très peu équipés pour pratiquer l'agriculture sur des lots de terres boisées. Il raconte ainsi une visite au bureau de l'administrateur des secours directs à Newcastle, où il a défendu avec succès une requête de son beau-frère Stanislas Morais :

J'ai dit, là, pour Stanislas, les enfants qu'il avait, puis... Il dit : « C'est un étranger, il dit, il appartient de Newcastle ? » Ah, j'ai dit : « Il appartient pas de Newcastle d'une manière mais, j'ai dit, il appartient de la famille. J'ai dit : « Ils avont mouvé, j'ai dit, ils avont rien fait, j'ai dit, ils restiont à Tilley Road », Tilley Road, qu'ils appeliont. J'ai dit : « Il a pas d'ouvrage, puis...

- Well, il dit, Kelly, il dit, we can 't give it to him. We're not allowed to.

- Ha! There's lots of things you're not allowed, j'ai dit, but you're doin 'it.

- Well, il dit, I wouldn't know a thing about that.

- Well anyway, j'ai dit...»

Après une escousse, j'ai dit : « Si tu peux rien faire, je vais aller voir la police »" ${ }^{10}$.

Alain Kelly semble avoir été accepté sans problèmes comme Acadien parce qu'il était parfaitement intégré dans les communautés acadiennes où il a vécu, qu'il s'agisse de Pointe-Sapin, de Tracadie ou de Beaver Brook. Il partageait la langue et la culture des habitants de ces endroits et il était très apprécié comme chanteur folklorique. Il raconte, par exemple, que pendant les années passées à Tracadie, il était souvent sollicité pour chanter lors des noces. Kelly s'est toutefois trouvé dans une position difficile à partir du moment

10. CÉA, coll. R. Labelle, enreg. 3549 (2000). 
où il est devenu commerçant à Beaver Brook en 1942. Il avait réussi à acheter un petit commerce grâce à l'appui d'un acheteur de bois anglophone qui voulait le faire servir d'intermédiaire auprès de la population. Il s'agit d'une rare occasion où son identité en partie anglophone l'a aidé économiquement. Lorsqu'il refusait de vendre à crédit à des habitants qu'il ne jugeait pas fiables, ces derniers le traitaient alors de « maudit Irlandais ». On l'accusait aussi de s'enrichir sur le dos des pauvres.

De telles accusations auraient probablement été lancées contre lui par des habitants jaloux peu importe son origine ethnique. Il y a cependant un fait qui a mis Alain Kelly dans une situation particulièrement difficile : c'est que le premier curé de la paroisse de Beaver Brook, Louis Lamontagne, affichait une attitude de méfiance envers lui. Le curé Lamontagne se donnait comme mission de protéger les colons de Beaver Brook contre les influences assimilatrices, étant donné qu'ils vivaient dans la seule paroisse francophone de la Miramichi. Il voyait donc Kelly comme un Irlandais dont il fallait limiter l'influence. Alain Kelly explique ainsi la situation :

Moi, je savais qu'il me regardait pas beaucoup, puis en même temps, j'étais pas derrière avec mes dîmes. Je payais tout le tèmps mes dîmes, puis je les aidais pour les pique-niques, puis toutes sortes d'affaires de même, hein. Je sais pas, on dirait qu'il aimait pas l'Irlandais. Oui, puis je parlais aussi bien français comme lui $^{11}$.

Il est difficile de comprendre l'attitude négative du curé, compte tenu du fait qu'Alain Kelly était francophone et élevait sa famille en français. On peut supposer que le curé voyait les personnes bilingues d'un mauvais œil, puisqu'en milieu minoritaire, le bilinguisme peut être un pas vers l'anglicisation. Il y avait à Beaver Brook des colons acadiens qui avaient adopté la langue anglaise après avoir vécu pendant plusieurs années dans des communautés anglophones de la Miramichi, comme Newcastle, Chatham et Chatham Head. Ces derniers pouvaient donc s'adresser à Alain Kelly en anglais, ce que déplorait le curé Lamontagne, qui désirait refranciser les Acadiens en voie d'assimilation. Le fait que Kelly exploitait un commerce en plein cœur de Beaver Brook pouvait aussi inquiéter le curé, qui craignait sans doute que l'anglais y soit la langue dominante. Il est possible aussi que Louis Lamontagne était avant tout méfiant envers les Irlandais en général, ou encore qu'il considérait Kelly comme un rival, lui qui se voyait comme l'unique chef dans la communauté.

On voit donc qu'un individu comme Alain Kelly pouvait être perçu de différentes façons, selon le point de vue que l'on avait : les Acadiens de son

11. CÉA, coll. R. Labelle, enreg. 2938 (1994). 
milieu le considéraient comme l'un des leurs. Les bûcherons anglophones qu'il avait côtoyés le voyait comme un « Irlandais français », donc un individu avec une double identité culturelle, parce qu'il s'identifiait aux travailleurs acadiens malgré son nom de famille. Le curé Lamontagne, pour sa part, le considérait tout simplement comme un Irlandais parce qu'il s'appelait Kelly et parlait couramment anglais.

Alain et Léontine Kelly ont eu onze enfants entre 1925 et 1945, dont trois sont décédés en bas âge. Parmi les huit autres, quatre sont nés à Tracadie et quatre à Beaver Brook. Ils ont tous été élevés en français, mais aucun n'est demeuré à Beaver Brook. L'effort de coloniser ce district pendant les années 1930 a été un demi-échec et les enfants des Kelly sont partis l'un à la suite de l'autre, pour vivre soit en Ontario, à Halifax ou dans diverses communautés de la Miramichi. Ceux et celles qui ont eu des enfants à leur tour les ont élevés en anglais, puisqu'ils vivaient dans des milieux anglophones. Aucun ne semble avoir fait un effort pour transmettre le français à la génération suivante. Alain et Léontine Kelly eux-mêmes ont déménagé à Newcastle pendant les années 1960 et alors qu'ils vivaient dans un quartier à forte proportion francophone, ils déploraient le fait que la plupart des Acadiens de leur entourage préféraient s'exprimer en anglais.

À Newcastle et ailleurs dans la Miramichi, il existe toujours un sentiment anti-français. Bien que la situation ait changé quelque peu depuis l'ouverture d'un centre scolaire et communautaire francophone dans la région en 1986, il reste que les Acadiens n'ont pas tendance à affirmer leur culture ouvertement. Alain Kelly a toujours été à l'aise autant parmi les anglophones que les francophones, mais il accepte mal de voir des Acadiens parler anglais entre eux. Il peut paraitre ironique de voir un individu d'origine mixte défendre le français dans la Miramichi, alors que des personnes d'origine entièrement acadienne ne font pas d'effort en ce sens. Pour comprendre cela, il suffit de se rappeler qu'Alain Kelly lui-même n'a pas à craindre la discrimination envers les Acadiens, puisqu'il peut passer pour un Irlandais s'il le désire. On peut se surprendre du fait qu'il n'a pas choisi de se ranger du côté des anglophones tout au long de sa vie, car cela aurait pu lui être bénéfique au point de vue économique et social. Il semble donc que l'influence de la culture acadienne dans son milieu d'origine à Pointe-Sapin a été déterminante pour lui : il aurait pu, bien sûr, devenir un «Irlandais » dans la Miramichi, mais il ne s'y serait jamais senti tout à fait à l'aise.

La participation d'Alain Kelly au Miramichi Folksong Festival nous fournit une bonne illustration des rapports complexes qu'il entretenait avec la population anglophone. Possédant un vaste répertoire de chansons folkloriques, tant en français qu'en anglais, Kelly s'est montré intéressé à 
participer au festival dès son inauguration en 1958. Pendant les premières années du festival, environ le tiers des chanteurs étaient acadiens, mais cette proportion a diminué rapidement et Kelly a graduellement intégré des chansons anglaises dans son répertoire pour se conformer au caractère de plus en plus anglophone de l'événement. Il est intéressant de noter qu'il y a même interprété des traductions anglaises de chansons qu'il avait d'abord apprises en français ${ }^{12}$. Par exemple, il a préparé une version anglaise de la complainte acadienne appelée Xavier Gallant ou Le meurtrier de sa femme, une chanson qui relate un meurtre commis à l'Île-du-Prince-Édouard en $1812^{13}$.

Bien que les chansons françaises fussent acceptées au Miramichi Folksong Festival, l'événement mettait l'accent sur les chants des bûcherons anglophones, ce que Kelly ressentait clairement. Il est significatif que le recueil intitulé Songs of Miramichi, publié par Louise Manny en 1968, ne comprenne aucune chanson française ${ }^{14}$. Cet ouvrage est considéré comme une véritable anthologie de chansons de la Miramichi. Dans l'introduction, Manny écrit au sujet de ses années de cueillette :

Little did I think what lay before me ! [...] That I would meet Wilmot MacDonald who knows a hundred songs... That Sarah Ginnish would come down from Eel Ground and sing five songs in Micmac... That the completely bilingual Allan Kelly would sing French and English songs, among the latter the ancient False Knight upon the Road ${ }^{15}$.

Kelly a toujours tenu à interpréter au moins une chanson française à chacune de ses participations au Miramichi Folksong Festival. Après avoir chanté au moins cinq chansons en anglais, il clôturait ordinairement sa performance avec une chanson française qu'il introduisait en anglais. Il commente ainsi son insistance à accorder une place à la chanson française :

Mon nom est irlandais, mais j'ai pas honte de parler français, je te garantis, icite, moi. Si ils voulont pas accepter mon français au festival, bien qu'ils s'arrangiont ! Je vais le chanter anyway ${ }^{16}$.

Au cours des dernières années, Kelly a choisi de redonner une place de choix à son répertoire français. Par contraste, les autres chanteurs locaux, qui présentent chaque année une image traditionnelle de la Miramichi à travers la chanson, s'expriment maintenant tous en anglais. Son épouse Léontine est

12. Certaines traductions provenaient d'un ouvrage de Marius Barbeau, Folk-songs of Old Québec, Ottawa, Musée national du Canada, 1962.

13. Voir Georges Arsenault, Complaintes acadiennes de l'̂lle-du-Prince-Édouard, Montréal, Leméac, 1980, pp. 117-168.

14. Louise Manny et James Reginald Wilson, Songs of Miramichi, Fredericton, Brunswick Press, 1968.

15. Ibid., p. 15.

16. CÉA, collection R. Labelle, enreg. 112 (1979). 
montée sur scène deux fois pour accompagner son mari dans des duos, mais n'aurait pas osé chanter seule, puisqu'elle n'était pas identifiée comme irlandaise et ne se sentait donc pas acceptée comme une représentante de la région, bien qu'elle y eût passé près de soixante ans de sa vie. Une des filles d'Alain et Léontine Kelly a aussi chanté à plusieurs reprises au festival, mais toujours en anglais.

Le fait que les enfants d'Alain Kelly n'aient pas cherché à maintenir leur identité acadienne indique encore une fois l'influence du milieu sur la culture. Même s'ils ont été élevés dans le village francophone de Beaver Brook, ils ont ressenti des pressions assimilatrices en vivant dans la Miramichi. Tout comme Alain Kelly est devenu Acadien à cause de l'environnement dans lequel il a vécu à Pointe-Sapin, ses enfants sont devenus anglophones dans la Miramichi. Mon impression est donc qu'un individu qui partage deux cultures ne choisit pas pleinement celle à laquelle il veut s'identifier, mais qu'il a tendance à aller du côté de celle qui exerce le plus de pression sur lui.

Si l'on retrace le parcours de la famille Kelly, on s'aperçoit que William Kelly, unilingue anglophone, après avoir épousé une Acadienne à PointeSapin, a élevé des enfants qui se sont intégrés à la communauté acadienne. Son fils Alain a épousé une Acadienne à son tour et a élevé ses enfants en français, mais ces derniers se sont plus tard intégrés à la culture anglophone de la Miramichi. La génération suivante est maintenant composée d'unilingues anglophones.

Ceci démontre à quel point l'assimilation à la culture environnante peut être rapide : après être brièvement devenue acadienne, une famille Kelly du Nouveau-Brunswick est redevenue anglophone. Heureusement, Alain Kelly, celui qui avait été marqué par la culture acadienne qui l'a entourée à PointeSapin et dans les chantiers forestiers du Nouveau-Brunswick, a si bien assimilé cette culture qu'il est devenu l'un des meilleurs représentants de la tradition musicale acadienne, ainsi que des coutumes et croyances de ce peuple ${ }^{17}$. Les archives de folklore du Centre d'études acadiennes en sont grandement enrichies. C'est d'ailleurs un phénomène surprenant, mais pourtant assez courant, de voir un individu un peu marginal par rapport à son groupe culturel devenir un représentant par excellence des traditions de sa communauté. Un exemple à mentionner serait le regretté Émile Benoît, un membre de la petite minorité française de la côte ouest de Terre-Neuve, qui est devenu l'ambassadeur musical de sa province pendant les années 1980 et 1990.

Enfin, je constate que les membres de la famille Kelly ont été influencés par la culture dominante à chaque endroit où ils ont vécu. À Pointe-Sapin et

17. Voir Ronald Labelle, «J'avais le pouvoir d'en haut : La représentation de l'identité dans le témoignage autobiographique d'Alain Kelly », thèse, Ph.D., Université Laval, 2001. 
à Tracadie, la culture acadienne francophone a exercé une profonde influence sur eux, alors que, dans la Miramichi, les influences anglophones ont fini par redonner un caractère irlandais aux Kelly. Une telle conclusion suggère évidemment que l'on est à la merci des influences de son milieu, auxquelles on ne peut résister. Frederik Barth énumère cependant différentes stratégies employées par des membres de groupes minoritaires en contact avec une majorité dominante. Certaines stratégies mènent à l'assimilation ou à une marginalisation. Il mentionne cependant qu'un groupe qui met l'accent sur son identité ethnique et qui s'organise pour l'affirmer par diverses activités peut maintenir sa vitalité ${ }^{18}$.

Les Acadiens vivant dans la Miramichi ont aujourd'hui les outils nécessaires pour s'affirmer. Reste à voir si ces outils seront suffisants pour contrer les influences assimilatrices du milieu. Mais il reste aussi à voir si les membres de la communauté choisiront de résister à l'assimilation. Comme nous l'avons vu, Alain Kelly a choisi d'être et de demeurer Acadien, alors qu'il aurait pu opter pour une identité irlandaise et anglophone. Évidemment, les choix personnels qu'il a faits n'ont pas nécessairement eu une influence déterminante sur les autres personnes de son milieu.

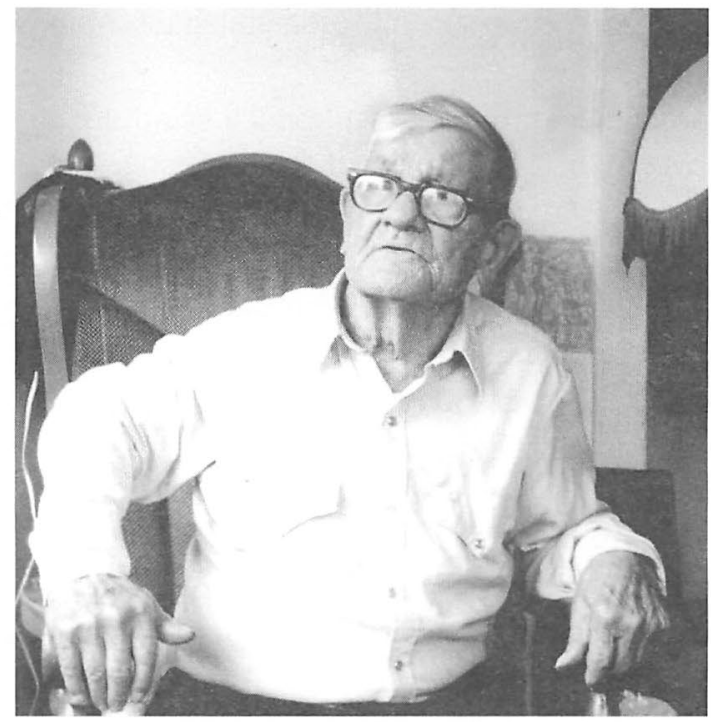

Alain Kelly chez lui en 1997

18. Frederick Barth, op. cit., p. 33. 